\title{
Pest Control in Agricultural Plantations Using Image Processing
}

\author{
Murali Krishnan ${ }^{1}$; Jabert. $G^{2}$ \\ 1. M.E.Communication Systems (Electronics and Communication),Bannari Amman Institute of Technology.
}

\begin{abstract}
Monocropped plantations are unique to India and a handful of countries throughout the globe. Essentially, the FOREST approach of growing coffee along with in India has enabled the plantation to fight many outbreaks of pests and diseases. Mono cropped Plantations are under constant threat of pest and disease incidence because it favours the build up of pest population. To cope with these problems, an automatic pest detection algorithm using image processing techniques in MATLAB has been proposed in this paper. Image acquisition devices are used to acquire images of plantations at regular intervals. These images are then subjected to pre-processing, transformation and clustering.
\end{abstract}

\section{Introduction}

Today there are more than 250 organic pesticides and thousands of formulations. The coffee industry unfortunately relies on these poisons to protect the plant and berries from insect attack and disease spread. In some advanced countries aerial spraying of these hazardous chemicals is carried out to save on labour costs. Most, coffee farmers advocate the use of BROAD SPECTRUM pesticides. These are more dangerous than systemic pesticides because they act on many insects both beneficial and harmful. There is every chance that these chemicals can easily drift or get washed or leached by heavy showers and reach groundwater or open estuaries there by contaminating the earth's precious water reserve. However, the cultivation of these crops for optimum yield and quality produce is highly technical. A lot of research has been done on greenhouse agro systems

And more generally on protected crops to control pests and diseases by biological means instead of pesticides. Research in agriculture is aimed towards increase of productivity and food quality at reduced expenditure and with increased profit, which has received importance in recent time. A strong demand now exists in many countries for non-chemical control methods for pests or diseases. However no automatic methods are available which precisely and periodically detect the pests on plants. In fact, in production conditions, greenhouse staff periodically observes plants and search for pests. This manual method is time consuming. With the recent advancement in image processing pattern recognition techniques, it is possible to develop an autonomous system for disease classification of crops.

In this paper, we focus on early pest detection. First, this implies to regularly observe the plants. Disease images are acquired using cameras or scanners. Then the acquired image has to be processed to interpret the image contents by image processing methods. The focus of this paper is on the interpretation of image for pest detection.

\section{Image Segmentation}

In computer vision, segmentation is the process of partitioning a digital image into multiple segments (sets of pixels, also known as super pixels). The goal of segmentation is to simplify and/or change the representation of an image into something that is more meaningful and easier to analyse. Image segmentation is typically used to locate objects and boundaries (lines, curves, etc.) in images. More precisely, image segmentation is the process of assigning a label to every pixel in an image such that pixels with the same label share certain visual characteristics.

The result of image segmentation is a set of segments that collectively cover the entire image, or a set of contours extracted from the image. Each of the pixels in a region is similar with respect to some characteristic or computed property, such as colour, intensity, or texture.

\section{Clustering Method}

This is an iterative technique that is used to partition an image into clusters. Procedure of clustering method is explained in figure 2.1. Clusters can be selected manually, randomly, or based on some conditions. Distance between the pixel and cluster centre is calculated by the squared or absolute difference between a pixel and a cluster centre.

Clusters can be selected manually, randomly, or based on some conditions. Distance between the pixel and cluster centre is calculated by the squared or absolute difference between a pixel and a cluster centre. The difference is typically based on pixel colour, intensity, texture, and location, or a weighted combination of these 
factors. More commonly used clustering algorithms are K - means algorithm, fuzzy c-means algorithm, expectation - maximization (EM) algorithm. The quality of the final result of the clustering method depends mainly on the initial set of clusters. Since the algorithm is extremely fast, a collective method is to run the algorithm several times and select the best clustering obtainable.

Fig1.Flow chart for clustering Algorithm

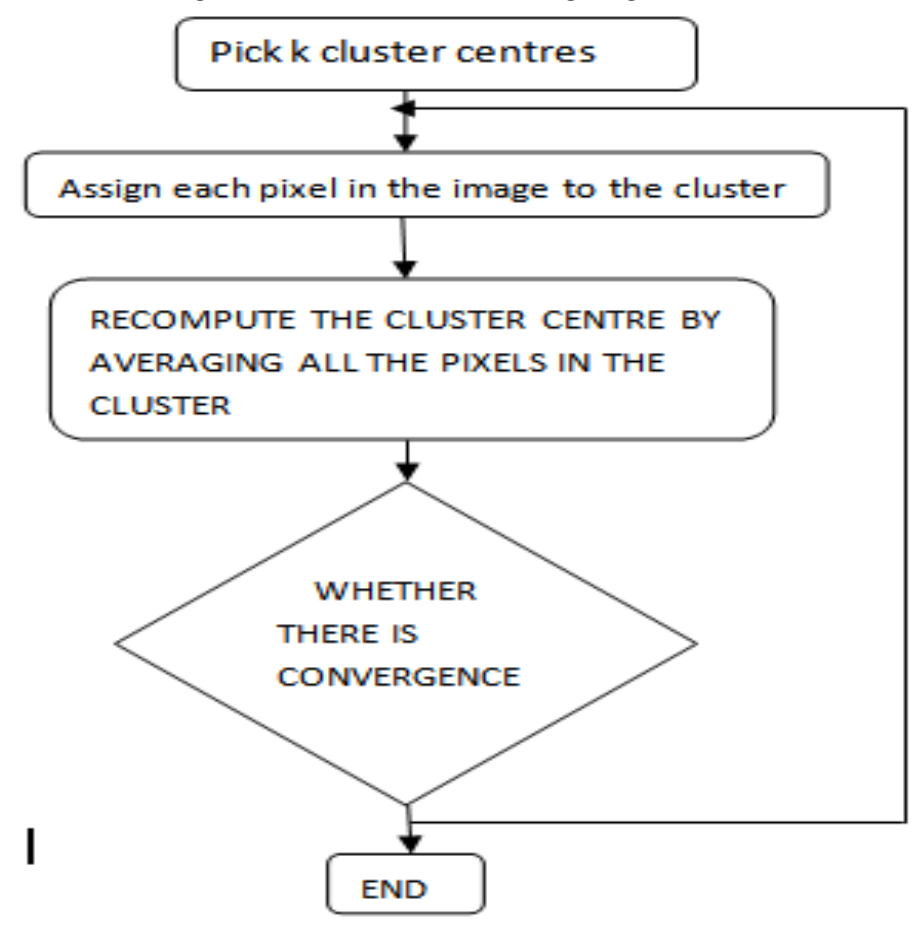

A drawback of the clustering algorithm is that the number of clusters $\mathrm{k}$ is an input parameter. A wrong choice of $\mathrm{k}$ may yield poor results. The algorithm also assumes that the variance is an appropriate measure of cluster scatter. The following method can be employed to find the cluster centres.

1. Compute the intensity distribution (also called the histogram) of the intensities.

2. Initialize the centroids with $\mathrm{k}$ random intensities.

$\mathrm{C}(\mathrm{i})=\operatorname{argminj}\|\mathrm{X}(\mathrm{i})-\mu \mathrm{j}\|^{2}$

3. Cluster the points based on distance of their intensities from the centroid intensities.

4. Compute the new centroid for each of the clusters.

Where $\mathrm{k}$ is a parameter of the algorithm (the number of clusters to be found), $\mathrm{i}$ iterates over the all the intensities, $\mathrm{j}$ iterates over all the centroids and $\mu \mathrm{i}$ are the centroid intensities. The $\mathrm{k}$-means clustering was invented in 1956. The most common form of the algorithm uses an iterative refinement heuristic known as Lloyd's algorithm. Since the algorithm is extremely fast, a common method is to run the algorithm several times and return the best clustering found.

\section{ADVANTAGES OF k-means clustering}

In particular when using heuristics such as Lloyd's algorithm is rather easy to implement and apply even on large data sets. As such, it has been successfully used in various topics, ranging from market segmentation, computer vision, geo-statistics to agriculture. It often is used as a pre-processing step for other algorithms. For example, to find a starting configuration. Other existing methods for image segmentation are compression based, histogram based, region growing methods, edge detection, split and merge methods, based on partial differential equations etc.

\section{Proposed Method}

The segmentation algorithm alone cannot provide good quality output, it needs pre-processing step. Pre-processing may consist of various steps like de-noising and image enhancement. De-noising provided with the rank filter, which well suited for the pest image. It is non-linear filter, which preserve the shape, edge and other information without lack of clarity (Krit somkanth, 2011), (R M Hodgson 1985). Due to the irregularities and drawbacks in pest images, it is mandatory to include the pre-processing step before the segmentation process for the quality and accurate output. The proposed method is shown in figure 2 . 


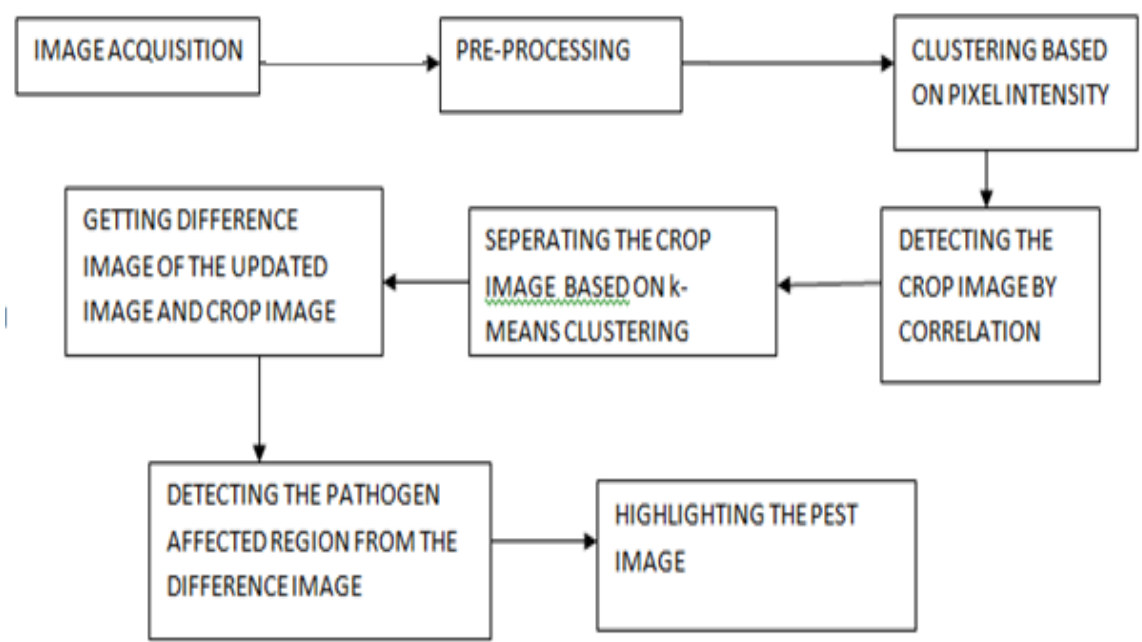

Fig.2.block diagram for the proposed method

In the proposed method, pre-processing is added before the segmentation and volume estimation is added after the segmentation. Also an image subtraction step is added after the algorithm. Proposed method reduces the problems due to noises and other irregularities in acquired images and also it overcome the disadvantages like intensity in homogeneity and artefacts, etc.

\section{Experimental Results And Discussion}

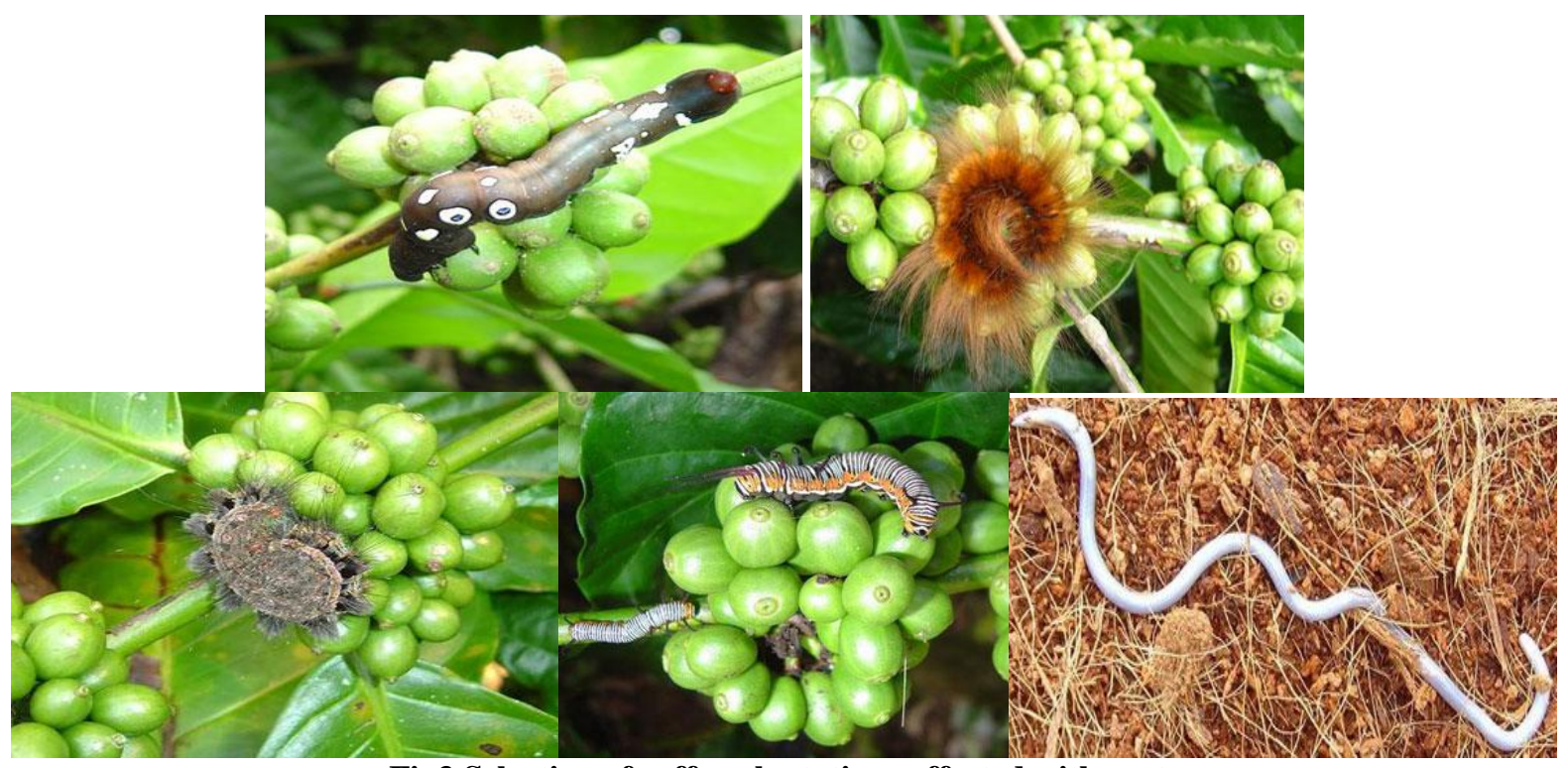

Fig3.Selection of coffee plantations affected with pests

The figure 3 shows the ROI selection of a infarction region. This selection helps to analyse the needed region alone. In this method, the pixels inside the rectangular mask alone are taken and the remaining pixels are left. It done by regularly capturing the field Images. 


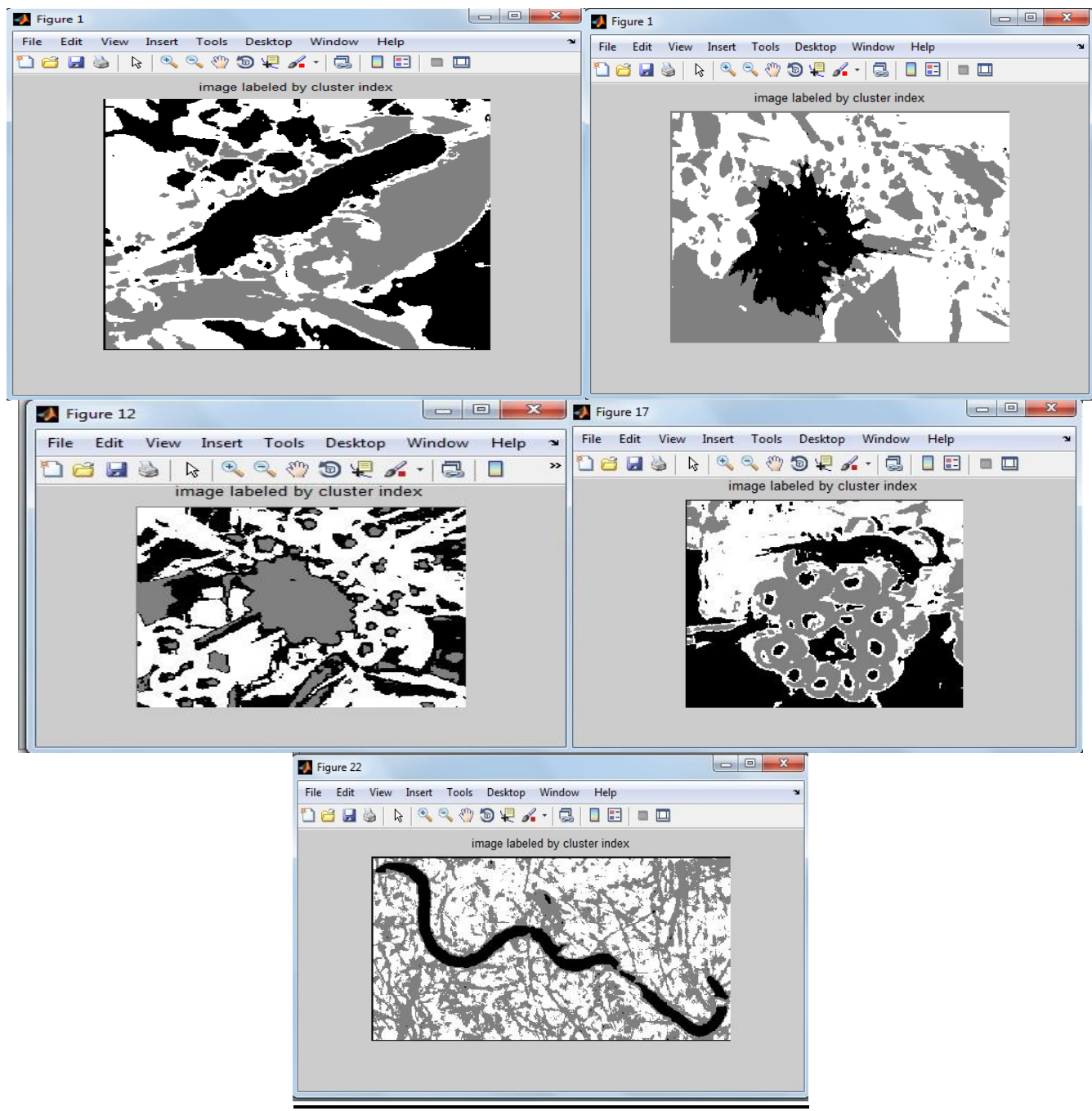

Fig.4. clustering based on intensity and threshold seperation

\section{Clustering To Separate Plant And Pest}

Masking and Removing green pixels: Masking means setting the pixel value in an image to zero or some other background value. In this step, we identify mostly the green coloured pixels. After that, based on specified threshold value that is computed for these pixels. The green components of the pixel intensities are set to zero if it is less than the pre-computed threshold value. Then red, green and blue components of this pixel is assigned zero value by mapping RGB components. The green coloured pixels mostly represent the healthy areas of the leaf and they do not add any valuable weight to disease identification .

Segmentation: From the above steps, the infected portion of the leaf is extracted. The infected region is then segmented into a number of patches of equal size. In this approach patch size of $32 X 32$ is taken.

Obtaining Useful Segments: In this step the useful segments are obtained. The size of the patch is chosen in such a way that the significant information is not lost. Not all segments contain significant amount of information. So the patches which are having more than fifty percent of the information are taken into account for the further analysis. 


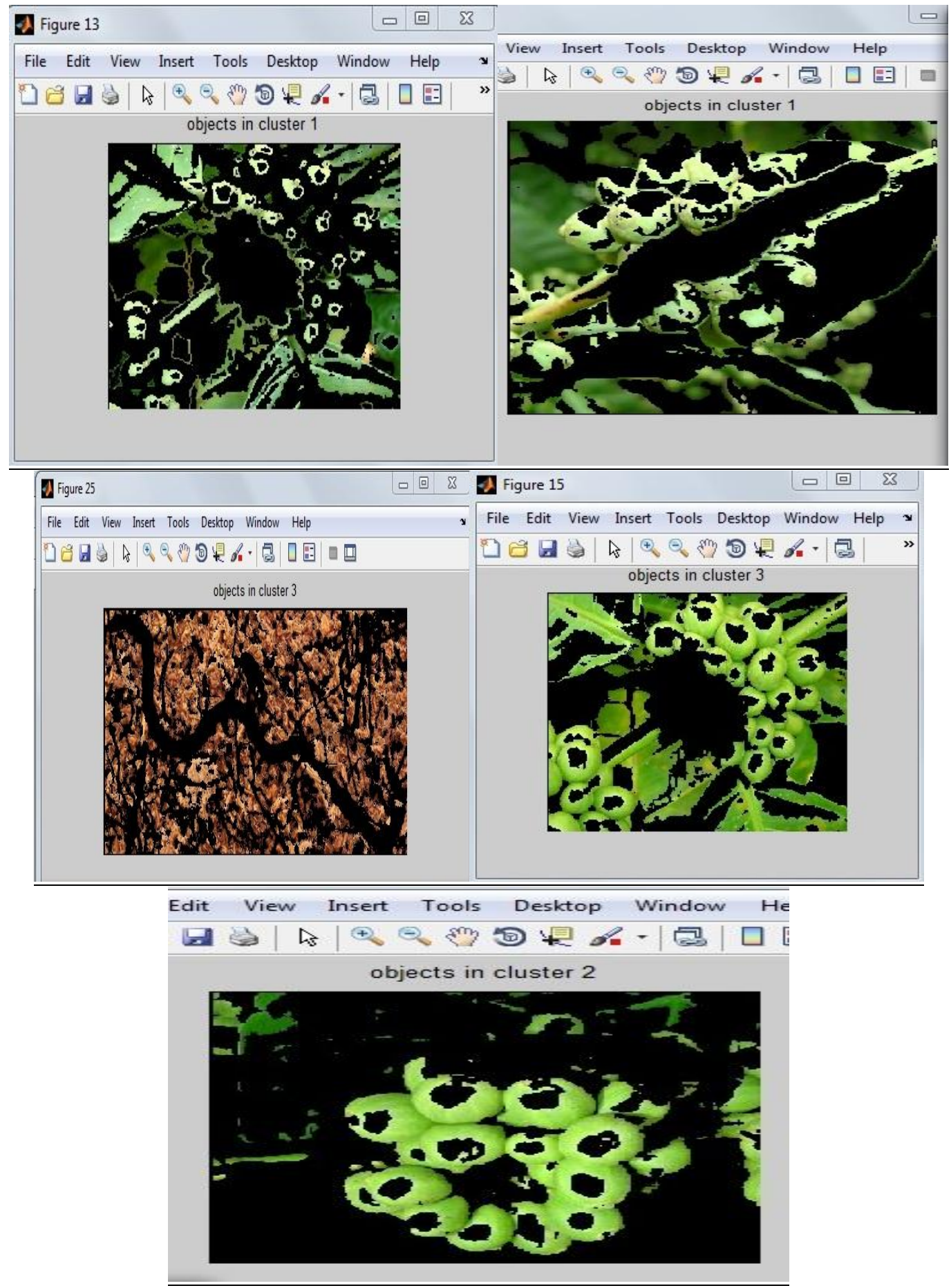

Fig5. clustering to separate plant areas 


\section{Final Clustering To Separate Pest Images Based On Image Subtraction}

The final clustering called differential clustering is done by subtracting the clustered plant image from the acquired image. Thus from the difference image, we obtain the pest image as shown .

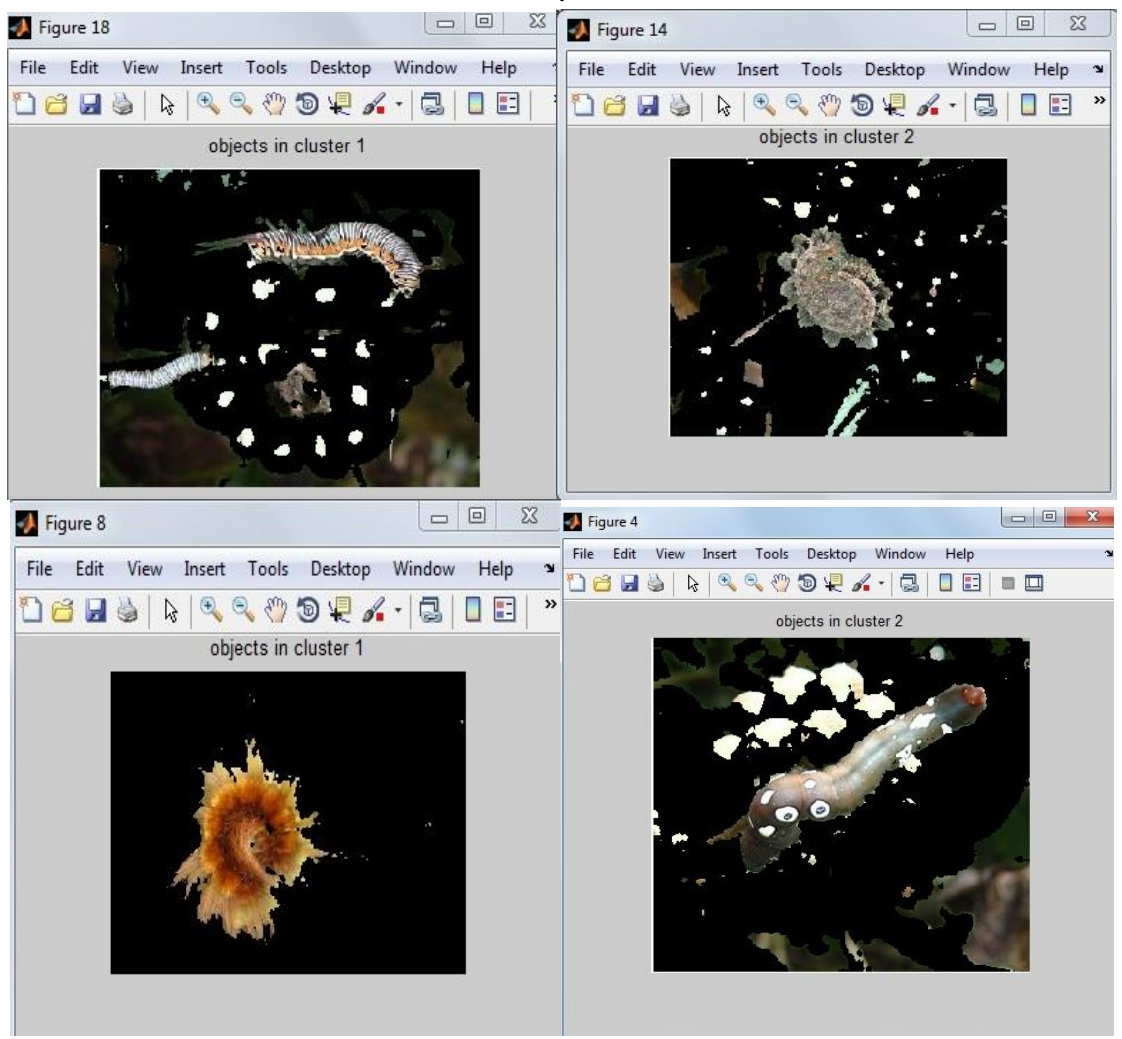

Fig6.final clustered image of the pest

\section{Conclusion}

The use of Artificial insecticides and nematicides have been degrading the quality of plantation crops for many years. In this paper a novel algorithm is presented for easily identifying the pest infected areas of these crops. The algorithm can be further modified for finding the diseased areas in the crops by using sophisticated softwares and better image acquisition devices. All over the world agriculture experts are working on eradication of bioagressors and infected coffee plantation are one of the challenges out of it. Image Processing technique plays a vital role in it. Our first objective is to detect diseases like coffee berry on plantations and other bioagressors (aphids) or plant diseases . Cognitive approach introduce new objects to detect or new image processing programs to extract the corresponding information. We propose an original approach for early detection of the of pest on crops. To detect biological objects on a complex background, we combined scanner image acquisition, sampling optimization, and advanced cognitive vision. It illustrates the collaboration of complementary disciplines and techniques, which led to an automated, robust and versatile system. The prototype system proved reliable for rapid detection of pests. It is rather simple to use and exhibits the same performance level as a classical manual approach. Our goal is rather to better spot the starting points of bioagressors attacks and to count these so that necessary action can be taken.

\section{Refrences}

[1]. Gonzalez, R., R. Woods and S. Eddins, 2004. Digital Image Processing using MATLAB. 1st Edn., Printice Hall, ISBN: 0130085197 ,pp: 624.

[2]. W. Petry and W. Kuhbauch, "Automated discrimination of weed species with quantitative image analysis," Journal of Agronomy \& Crop Science, vol. 163, pp. 345-351, 1989.

[3]. R.D. Tillet, "Image analysis for agricultural processes: a review of potential opportunities," Journal of Agricultural Engineering Research, vol. 12, pp. 247-258, 1991.

[4]. J. Hemming and T. Rath, "Computer-vision based weed identification under field Journal of Agricultural Engineering Research, vol. 78- 3, pp. 233-243, March 2001

[5 ]. ALFANO, J. R. (2009). Roadmap for future research on plant pathogen effectors. Molecular Plant Pathology 10,805813.

[6]. ALFANO, J. R. \& COLLMER, A. (2004). Type III secretion system effector proteins: double agents in bacterial diseaseand plant defence. Annual Review of Phytopathology 42,385-414.

[7]. Alexander, M. 1977. Introduction to soil Microbiology. 2nd edition. New York. John Wiley and sons. 
[8]. Alexander, M. 1974. Microbial Ecology. New York. John Wiley and sons.

[9]. Baker. E. F and R.J. Cook, 1974. Biological control of plant pathogens. W.H. Freeman \& Co. Sanfransisco, 433pp.

[10]. Baker. E. F. 1987. Evolving concepts of biological control of plant pathogens. Annul. Rev. Phytopathol., 25: 67-85.

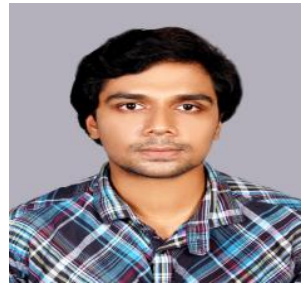

Murali Krishnan received his B-Tech degree in Electronics and Communications Engineering from Government Engineering College, Attingal Kerala under Cochin University of science and technology (2006-10).He is currently acquiring his M.E post graduation in Communication Systems from Bannari Amman Institute of Technonlogy, Sathyamangalam. 